\title{
Initial Value Problems for Integrable Systems on a Semi-Strip
}

\author{
Alexander L. SAKHNOVICH \\ Vienna University of Technology, Institute of Analysis and Scientific Computing, \\ Wiedner Hauptstr. 8, A-1040 Vienna, Austria \\ E-mail: oleksandr.sakhnovych@tuwien.ac.at
}

Received September 01, 2015, in final form December 28, 2015; Published online January 03, 2016 http://dx.doi.org/10.3842/SIGMA.2016.001

\begin{abstract}
Two important cases, where boundary conditions and solutions of the well-known integrable equations on a semi-strip are uniquely determined by the initial conditions, are rigorously studied in detail. First, the case of rectangular matrix solutions of the defocusing nonlinear Schrödinger equation with quasi-analytic boundary conditions is dealt with. (The result is new even for a scalar nonlinear Schrödinger equation.) Next, a special case of the nonlinear optics ( $N$-wave) equation is considered.
\end{abstract}

Key words: Weyl-Titchmarsh function; initial condition; quasi-analytic functions; system on a semi-strip; nonlinear Schrödinger equation; nonlinear optics equation

2010 Mathematics Subject Classification: 35Q55; 35Q60; 34B20; 35A02

\section{Introduction}

Cauchy problems for wave equations were successfully investigated using the classical inverse scattering transform, Laplace transforms and also some other methods. The theory of initialboundary value problems (and problems in a quarter-plane or semi-strip) is somewhat more complicated even for the case of linear wave equations. Some results, discussions and references on this topic are given in $[3,16,19,22,36,45]$. The mentioned above results and discussions are also related to the integrable nonlinear equations. Although it is impossible to refer here to the whole variety of important publications on the initial-boundary value problems for integrable wave equations, we would like to list just some: $[9,10,12,16,18,19,27,30,31,32,33,37,39$, 40, $43,48,50,55]$. Usually (excluding, e.g., sine-Gordon case [32, 37, 42, 48]), initial-boundary value problems are overdetermined (see $[11,18,48]$ ) for such cases of integrable nonlinear equations, where exact procedures to recover solutions from initial and boundary values exist. Thus, the reduction of the initial-boundary conditions, which are necessary to recover solutions, becomes crucial for solving initial-boundary value problems.

In this paper we extend and develop further the work (which was started in [47]) on the reduction of the necessary initial-boundary conditions. Namely, a case, where boundary conditions provide direct information about an initial condition, was investigated in [47]. Here we study the cases, where an initial condition provides direct information about boundary conditions, and the solutions of the well-known nonlinear integrable equations in a semi-strip are uniquely defined by the initial conditions. (One could speak, perhaps, about additional symmetries of the solutions.)

We consider such situations using the inverse spectral transform approach [6, 7, 28]. More precisely, we follow the scheme introduced in [49, 51], see also [52, Chapter 12] and references therein. That is, we describe the evolution of Weyl-Titchmarsh (Weyl) function in terms of the linear-fractional transformations. The scheme is applicable to various integrable equations and several interesting uniqueness and existence theorems were proved in this way (see [48, 
Chapter 6] and references therein for more details). Most of the mentioned above uniqueness and existence theorems were obtained for the equations with scalar solutions. Here we consider the matrix defocusing nonlinear Schrödinger (defocusing NLS or dNLS) equation

$$
2 v_{t}=\mathrm{i}\left(v_{x x}-2 v v^{*} v\right), \quad v_{t}:=\frac{\partial}{\partial t} v
$$

which is equivalent $[59,60]$ to the compatibility condition

$$
G_{t}-F_{x}+[G, F]=0 \quad[G, F]:=G F-F G
$$

of the auxiliary linear systems

$$
y_{x}=G y, \quad y_{t}=F y,
$$

where

$$
\begin{aligned}
& G=\mathrm{i}(z j+j V), \quad F=-\mathrm{i}\left(z^{2} j+z j V-\left(\mathrm{i} V_{x}-j V^{2}\right) / 2\right), \\
& j=\left[\begin{array}{cc}
I_{m_{1}} & 0 \\
0 & -I_{m_{2}}
\end{array}\right], \quad V=\left[\begin{array}{cc}
0 & v \\
v^{*} & 0
\end{array}\right],
\end{aligned}
$$

$I_{m_{1}}$ is the $m_{1} \times m_{1}$ identity matrix and $v$ is an $m_{1} \times m_{2}$ matrix function. We will consider dNLS equation on the semi-strip

$$
\mathcal{D}=\{(x, t): 0 \leq x<\infty, 0 \leq t<a\},
$$

and we note that the auxiliary system

$$
y_{x}=G y=\mathrm{i}(z j+j V) y
$$

is (for each fixed $t$ ) a well-known self-adjoint Dirac system, also called AKNS or ZakharovShabat system. Without changes in notation, we speak about usual derivatives inside domains and about left or right (which should be clear from the context) derivatives on the boundaries, and boundaries of $\mathcal{D}$ in particular. We should mention that, in the usual PDE setting, solutions are often considered in open domains but, in view of certain regularity of the solutions treated in our paper, the boundaries are included in $\mathcal{D}$ in our case.

Since $v$ in dNLS (1.1) is an $m_{1} \times m_{2}$ matrix function, interesting matrix, vector and multicomponent dNLS equations from [2, Chapter 4] are included in the considered class.

Another equation that we study in this paper is the nonlinear optics (or $N$-wave) equation:

$$
\begin{aligned}
& {\left[D, \frac{\partial \varrho}{\partial t}\right]-\left[\widehat{D}, \frac{\partial \varrho}{\partial x}\right]=[D, \varrho][\widehat{D}, \varrho]-[\widehat{D}, \varrho][D, \varrho], \quad \varrho=\varrho^{*},} \\
& D=\operatorname{diag}\left\{d_{1}, d_{2}, \ldots, d_{m}\right\}, \quad d_{1}>d_{2}>\cdots>d_{m}>0, \quad[D, \varrho]:=D \varrho-\varrho D,
\end{aligned}
$$

where $\varrho(x, t)$ is an $m \times m$ matrix function, $\operatorname{diag}\left\{d_{1}, d_{2}, \ldots\right\}$ stands for a diagonal matrix with the entries $d_{1}, d_{2}, \ldots$ on the main diagonal, and $\widehat{D}>0$ is another diagonal matrix.

First, we obtain the evolution of the Weyl function for the equation (1.8). Next, we consider an interesting special case, where (similar to the inequalities for the entries of $D$ ) we have

$$
\widehat{D}=\operatorname{diag}\left\{\widehat{d}_{1}, \widehat{d}_{2}, \ldots, \widehat{d}_{m}\right\}, \quad \widehat{d}_{1}>\widehat{d}_{2}>\cdots>\widehat{d}_{m}>0 .
$$

In Section 2 we formulate some necessary results on Weyl functions (and their evolution for the dNLS case), in Section 3 we recover the boundary conditions for dNLS from an initial 
condition on a semi-axis (and in this way we solve the initial value problem for dNLS in a semistrip), and Section 4 is dedicated to the evolution of the Weyl function and initial value problem in a semi-strip for the $N$-wave equation.

We note that the theory of Weyl functions (Weyl-Titchmarsh theory) is actively developing in recent years (see, e.g., [14, 15, 21, 23, 24, 35, 48, 52, 54, 56] and references therein) and its applications to initial-boundary value problems are of growing interest.

As usual, $\mathbb{R}$ stands for the real axis, $\mathbb{R}_{+}=(0, \infty), \mathbb{C}$ stands for the complex plane, and $\mathbb{C}_{+}$ for the open semi-plane $\{z: \Im(z)>0\}$. We say that $v(x)$ is locally summable if its entries are summable on all finite intervals of $[0, \infty)$. We say that $v$ is continuously differentiable if $v$ is differentiable and its first derivatives are continuous. The notation $\|\cdot\|$ stands for the $l^{2}$ vector norm or the induced matrix norm. The partial derivative $f_{x t}$ stands for $\partial f_{x} / \partial t$ and, correspondingly, $f_{t x}=\partial f_{t} / \partial x$.

\section{Preliminaries}

\subsection{Dirac system and dNLS}

We denote by $u$ the fundamental solution of system (1.7) normalized by the condition

$$
u(0, z)=I_{m}, \quad m=m_{1}+m_{2} .
$$

Definition 2.1. Let Dirac system $(1.7)$ on $[0, \infty)$ be given and assume that $V$ is locally summable. Then the Weyl function $\varphi$ is an $m_{2} \times m_{1}$ holomorphic matrix function, which satisfies the inequality

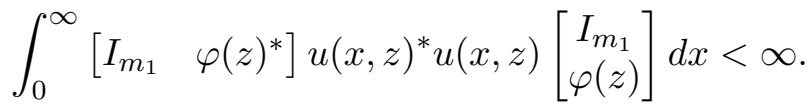

The following proposition is proved in [20] (see also [48, Section 2.2]).

Proposition 2.2. The Weyl function always exists and it is unique.

In order to construct the Weyl function, we introduce a class of $m \times m_{1}$ matrix functions $\mathcal{P}(z)$, which are an immediate analog of the classical pairs of parameter matrix functions. Namely, the matrix functions $\mathcal{P}(z)$ are meromorphic in $\mathbb{C}_{+}$and satisfy (excluding, possibly, a discrete set of points) the following relations

$$
\mathcal{P}(z)^{*} \mathcal{P}(z)>0, \quad \mathcal{P}(z)^{*} j \mathcal{P}(z) \geq 0, \quad z \in \mathbb{C}_{+} .
$$

It is said that $\mathcal{P}(z)$ are nonsingular (i.e., the first inequality in (2.2) holds) and with property- $j$ (i.e., the second inequality in (2.2) is valid). Relations (2.2) imply (see, e.g., [20]) that

$$
\operatorname{det}\left(\left[\begin{array}{ll}
I_{m_{1}} & 0
\end{array}\right] u(x, z)^{-1} \mathcal{P}(z)\right) \neq 0 .
$$

Definition 2.3. The set $\mathcal{N}(x, z)$ of Möbius transformations is the set of values (at the fixed $\left.x \in[0, \infty), z \in \mathbb{C}_{+}\right)$of matrix functions

$$
\varphi(x, z, \mathcal{P})=\left[\begin{array}{ll}
0 & I_{m_{2}}
\end{array}\right] u(x, z)^{-1} \mathcal{P}(z)\left(\left[\begin{array}{ll}
I_{m_{1}} & 0
\end{array}\right] u(x, z)^{-1} \mathcal{P}(z)\right)^{-1},
$$

where $\mathcal{P}(z)$ are nonsingular matrix functions with property- $j$.

Remark 2.4. It was shown in [20] that a family $\mathcal{N}(x, z)$, where $x$ increases to infinity and $z$ is fixed $\left(z \in \mathbb{C}_{+}\right)$, is a family of embedded matrix balls such that the right semi-radii are uniformly bounded and the left semi-radii tend to zero. (Recall that the $m_{2} \times m_{1}$ matrix ball, or Weyl matrix ball, with the center $\mathcal{M}$, the left semi-radius $\mathcal{R}_{l}$ and the right semi-radius $\mathcal{R}_{r}$ is the set of $m_{2} \times m_{1}$ matrices $\omega$ which may may be presented in the form $\omega=\mathcal{M}+\mathcal{R}_{l} \mathcal{U} \mathcal{R}_{r}$, where $\mathcal{U}$ are contractive $m_{2} \times m_{1}$ matrices.) 
Proposition 2.5 ([20]). Let Dirac system $(1.7)$ on $[0, \infty)$ be given and assume that $V$ is locally summable. Then, the sets $\mathcal{N}(x, z)$ are well-defined. There is a unique matrix function $\varphi(z)$ defined in $\mathbb{C}_{+}$and such that

$$
\{\varphi(z)\}=\bigcap_{x<\infty} \mathcal{N}(x, z)
$$

This function is analytic and non-expansive (i.e., contractive). Furthermore, this function coincides with the Weyl function of system (1.7).

Formula (2.3) is supplemented by the asymptotic relation

$$
\varphi(z)=\lim _{b \rightarrow \infty} \varphi_{b}(z)
$$

which is valid for any set of functions $\varphi_{b}(z) \in \mathcal{N}(b, z)$. Relation (2.4) follows from (2.3) and Remark 2.4 (see also [48, Remark 2.24]).

Next, we consider the famous compatibility condition (zero curvature equation) (1.2).

Proposition 2.6 ([45]). Let some $m \times m$ matrix functions $G$ and $F$ and their derivatives $G_{t}$ and $F_{x}$ exist on the semi-strip $\mathcal{D}$, let $G, G_{t}$ and $F$ be continuous with respect to $x$ and $t$ on $\mathcal{D}$, and let (1.2) hold. Then, we have the equality

$$
u(x, t, z) R(t, z)=R(x, t, z) u(x, 0, z), \quad R(t, z):=R(0, t, z),
$$

where $u(x, t, z)$ and $R(x, t, z)$ are normalized fundamental solutions given, respectively, by

$$
u_{x}=G u, \quad u(0, t, z)=I_{m} ; \quad R_{t}=F R, \quad R(x, 0, z)=I_{m} .
$$

The equality (2.5) means that the matrix function

$$
y(x, t, z)=u(x, t, z) R(t, z)=R(x, t, z) u(x, 0, z)
$$

satisfies on $\mathcal{D}$ both systems (1.3). Moreover, the fundamental solution u admits the factorization

$$
u(x, t, z)=R(x, t, z) u(x, 0, z) R(t, z)^{-1} .
$$

Proposition 2.6 and formula (2.4) yield [47] the following evolution theorem.

Theorem 2.7. Let an $m_{1} \times m_{2}$ matrix function $v(x, t)$ be continuously differentiable on $\mathcal{D}$ and let $v_{x x}$ exist. Assume that $v$ satisfies the $d N L S$ equation (1.1) as well as the following inequalities (for all $0 \leq t<a$ and some values $M(t) \in \mathbb{R}_{+}$):

$$
\sup _{x \in \mathbb{R}_{+}, 0 \leq s \leq t}\|v(x, s)\| \leq M(t) .
$$

Then, the evolution $\varphi(t, z)$ of the Weyl functions of Dirac systems (1.7) is given $($ for $\Im(z)>0)$ by the equality

$$
\varphi(t, z)=\left(R_{21}(t, z)+R_{22}(t, z) \varphi(0, z)\right)\left(R_{11}(t, z)+R_{12}(t, z) \varphi(0, z)\right)^{-1} .
$$

Remark 2.8. According to [46], the Dirac system $y_{x}=G y$ (where $G$ is given by (1.4) and (1.5) and $v$ is locally square summable) is uniquely recovered from the Weyl function $\varphi$. In other words, $v$ is uniquely recovered from $\varphi$, see the procedure in [46, Theorem 4.4]. The case of a more smooth (i.e., locally bounded) $v$ was dealt with in [48], see also references therein. 


\subsection{Auxiliary linear systems for nonlinear optics equation}

The nonlinear optics ( $N$-wave) equation (1.8) is the compatibility condition of the systems (1.3), where

$$
\begin{aligned}
& G(x, t, z)=\mathrm{i} z D-\zeta(x, t), \quad F(x, t, z)=\mathrm{i} z \widehat{D}-\widehat{\zeta}(x, t) \\
& \zeta=[D, \varrho], \quad \widehat{\zeta}=[\widehat{D}, \varrho] ;
\end{aligned}
$$

see [58] for the case $N=3$ and [1] for $N>3$. We shall need some preliminary results on the Weyl theory of the auxiliary system $y_{x}=G y$ from [48, Chapter 4] (see also [44]). The normalized fundamental solution $w$ of such a system is defined by the formula

$$
w_{x}(x, z)=(\mathrm{i} z D-\zeta(x)) w(x, z), \quad w(0, z)=I_{m}, \quad \zeta=-\zeta^{*} .
$$

Here and later we assume that $D$ is a fixed matrix satisfying (1.9). We consider system (2.10) with locally bounded potentials $\zeta$, that is, potentials satisfying (for each $l<\infty$ ) the inequality

$$
\sup _{0<x<l}\|\zeta(x)\|<\infty
$$

Definition 2.9. A generalized Weyl function (GW-function) of system (2.10), where $\zeta$ is locally bounded, is an $m \times m$ matrix function $\varphi$ such that for some $M>0$ it is analytic in the domain $\mathbb{C}_{M}^{-}=\{z: \Im(z)<-M\}$ and the inequality

$$
\sup _{x \leq l, \Im(z)<-M}\|w(x, z) \varphi(z) \exp \{-\mathrm{i} z x D\}\|<\infty
$$

holds for each $l<\infty$.

Remark 2.10. We note that the Weyl function of the system (2.10) is defined (for $\zeta$ bounded on $[0, \infty))$ by the analog $(2.17)$ of the inequality $(2.1)$ and by normalization conditions (2.18). If $\zeta$ is bounded on $[0, \infty)$, this Weyl function coincides with the normalized GW-function. (See the discussion after formula (2.18) and Definition 2.9 of the GW-function above.) The fact that the Weyl function satisfies (2.12) explains the term "generalized Weyl function" (or "GW-function").

The inverse spectral problem (ISpP) for system (2.10) is the problem of recovering (from a $\mathrm{GW}$-function $\varphi$ ) a potential $\zeta(x)=-\zeta^{*}(x)$ such that (2.12) is valid and the diagonal entries $\zeta_{k k}$ of $\zeta$ equal zero (i.e., $\left.\zeta_{k k} \equiv 0\right)$.

Notation 2.11. The notation $\mathfrak{M}$ stands for an operator mapping the pair $D$ and $\varphi$ into the corresponding potential $\zeta$ (i.e., $\mathfrak{M}(D, \varphi)=\zeta$ ). In other words, $\mathfrak{M}(D, \varphi)$ stands for a solution of the ISpP.

The following theorem (i.e., [48, Theorem 4.8]) is valid.

Theorem 2.12. For any matrix function $\varphi(z)$ which is analytic and bounded in $\mathbb{C}_{M}^{-}$and has the property

$$
\int_{-\infty}^{\infty}\left(\varphi(z)-I_{m}\right)^{*}\left(\varphi(z)-I_{m}\right) d \xi<\infty, \quad z=\xi+\mathrm{i} \eta, \quad \eta<-M
$$

there is at most one solution of the ISpP.

Our next corollary for systems on $(0, l)$ is immediate from the proof (see [48, pp. 108-109]) of Theorem 2.12. 
Corollary 2.13. Assume that (2.11) is valid and let relations (2.12) and (2.13) hold for an analytic and bounded matrix function $\varphi$. Then, $\zeta(x)$ is uniquely defined on $(0, l)$.

Remark 2.14. Under somewhat stronger (than in (2.13)) restrictions, the solution of the ISpP always exists. Namely, if for some matrix $\phi_{0}$ and some $M>0$ we have

$$
\begin{aligned}
& \sup _{\Im(z)<-M}\left\|z\left(\varphi(z)-I_{m}\right)\right\|<\infty, \quad \operatorname{det} \varphi(z) \neq 0 \quad \text { for } \Im(z)<-M, \\
& (\xi+\mathrm{i} \eta)\left(\varphi(\xi+\mathrm{i} \eta)-I_{m}-\phi_{0} /(\xi+\mathrm{i} \eta)\right) \in L_{m \times m}^{2}(-\infty, \infty) \text { for all fixed } \eta<-M,
\end{aligned}
$$

then $\mathfrak{M}(D, \varphi)$ is constructed in [48, Theorem 4.10].

The situation becomes simpler when $\zeta(x)$ is uniformly bounded on $[0, \infty)$, that is,

$$
\sup _{0<x<\infty}\|\zeta(x)\| \leq M_{0}
$$

We recall that a Weyl function of system (2.10) is introduced in another way than a GWfunction. Namely, a Weyl function is an analytic $m \times m$ matrix function $\varphi(z)$, satisfying for certain $M>0$ and $r>0$ and for all $z$ from the domain $\mathbb{C}_{M}^{-}=\{z: \Im(z)<-M\}$ the inequality

$$
\int_{0}^{\infty} \exp \{\mathrm{i} \bar{z} x D\} \varphi(z)^{*} w(x, z)^{*} w(x, z) \varphi(z) \exp \left\{-\left(\mathrm{i} z D+r I_{m}\right) x\right\} d x<\infty,
$$

and the normalization conditions on the entries $\varphi_{i j}(z)$ :

$$
\varphi_{i j}(z) \equiv 1 \quad \text { for } \quad i=j, \quad \varphi_{i j}(z) \equiv 0 \quad \text { for } \quad i>j .
$$

When (2.16) holds, a Weyl function of system (2.10) exists and is unique. Moreover, for that case $\varphi$ is the unique GW-function (of system (2.10) with the given $\zeta$ ) satisfying normalization conditions (2.18). In order to construct this Weyl (and simultaneously GW-) function we use matrices $j$ of the form (1.5) for each $1 \leq m_{1}<m$, that is, we set

$$
J_{k}:=\left[\begin{array}{cc}
I_{k} & 0 \\
0 & -I_{m-k}
\end{array}\right], \quad 1 \leq k<m .
$$

Now, the Weyl function is constructed [48, pp. 103-106] in the following way.

First, for each $k$, we introduce a class of $m \times(m-k)$ matrix functions $\mathcal{Q}_{k}$, which are meromorphic in some semi-plane $\mathbb{C}_{M_{k}}^{-}$and satisfy the inequalities

$$
\mathcal{Q}_{k}(z)^{*} \mathcal{Q}_{k}(z)>0, \quad \mathcal{Q}_{k}(z)^{*} J_{k} \mathcal{Q}_{k}(z) \leq 0,
$$

excluding, possibly, isolated points. These $\mathcal{Q}_{k}$ are called nonsingular with property- $J_{k}$. Assuming $M_{k}>2 M_{0} /\left(d_{k}-d_{k+1}\right)$ and using (2.19), one can show that the matrix function

$$
\psi_{k}(x, z)=\left[\begin{array}{ll}
I_{k} & 0
\end{array}\right] w(x, z)^{-1} \mathcal{Q}_{k}(z)\left(\left[\begin{array}{ll}
0 & I_{m-k}
\end{array}\right] w(x, z)^{-1} \mathcal{Q}_{k}(z)\right)^{-1}
$$

is well-defined for $x \geq 0, z \in \mathbb{C}_{M_{k}}^{-}$, and satisfies the inequality

$$
\left[\begin{array}{ll}
\psi_{k}(z)^{*} & I_{m-k}
\end{array}\right] J_{k}\left[\begin{array}{c}
\psi_{k}(z) \\
I_{m-k}
\end{array}\right] \leq 0, \quad \text { i.e., } \quad \psi_{k}(z)^{*} \psi_{k}(z) \leq I_{m-k} .
$$

The set of matrices $\psi_{k}(x, z)$ given by $(2.20)$, where $x$ and $z$ are fixed and matrix functions $\mathcal{Q}_{k}(z)$ are nonsingular with property- $J_{k}$, is denoted by $\mathcal{N}_{k}(x, z)$. These sets are embedded and have a point limit, that is, similar to (2.3) and (2.4) we have

$$
\breve{\psi}_{k}(z)=\bigcap_{x<\infty} \mathcal{N}_{k}(x, z), \quad \breve{\psi}_{k}(z)=\lim _{x \rightarrow \infty} \psi_{k}(x, z), \quad z \in \mathbb{C}_{M_{k}}^{-} .
$$


In this way we recover the $(k+1)$ th column of the Weyl function $\varphi$. More precisely, we have

$$
\left\{\varphi_{i, k+1}(z)\right\}_{i=1}^{k}=\breve{\psi}_{k}(z)\left[\begin{array}{c}
1 \\
0 \\
\ldots \\
0
\end{array}\right], \quad \Im(z)<-M
$$

for any $M>\max _{1 \leq k<m}\left(2 M_{0} /\left(d_{k}-d_{k+1}\right)\right)$. There is also an inverse transformation [48, Remark 4.6], which expresses $\breve{\psi}_{k}$ via $\varphi$ :

$$
\breve{\psi}_{k}(z)=\left[\begin{array}{ll}
I_{k} & 0
\end{array}\right] \varphi(z)\left[\begin{array}{c}
0 \\
I_{m-k}
\end{array}\right]\left(\left[\begin{array}{ll}
0 & I_{m-k}
\end{array}\right] \varphi(z)\left[\begin{array}{c}
0 \\
I_{m-k}
\end{array}\right]\right)^{-1}
$$

Finally, we will need a representation of $w(x, z)$ on intervals $0 \leq x \leq l, l<\infty$, [48, equation (4.37)] :

$$
w(x, z)=\exp \{\mathrm{i} z x D\}+\int_{d_{m} x}^{d_{1} x} \exp \{\mathrm{i} z t\} N(x, t) d t, \quad \sup _{x \leq l}\|N(x, t)\|<\infty .
$$

\section{NLS with quasi-analytic boundary conditions}

First publications on initial-boundary value problems for integrable systems (see, e.g., [28, 32]) appeared only several years after the great breakthrough for Cauchy problems for such systems. Interesting numerical [13], uniqueness [10, 57] and local existence [27, 37] results followed. Special linearizable cases of boundary conditions were found using symmetrical reduction [55] or BT (Bäcklund transformation) method [9, 25]. Global existence results for Dirichlet and Neumann initial-boundary value problems (for cubic NLS equations) were obtained using PDE methods in [12] and [29], respectively. Interesting approaches were developed by D.J. Kaup and H. Steudel [33], by P. Sabatier (elbow scattering) [39, 40] and by A.S. Fokas (global relation method) [18], see also some discussions on the corresponding difficulties and open problems in $[3,11]$. Since many publications were dedicated to the initial-boundary value problems for NLS equations, it is of special interest that a wide class of solutions of NLS in a semi-strip is uniquely determined by the initial condition.

We note that the case of quasi-analytic boundary (or initial) conditions is important also because related suggestions that initial and boundary conditions (or even solutions) belong to the so-called Schwartz class of functions are often used for simplicity (see, e.g., [17]). Our result shows that one should be rather careful with such suggestions, so that they agree with the established interrelations between initial and boundary conditions.

Recall that the domain $\mathcal{D}$ is defined in (1.6).

Notation 3.1. We consider $m_{1} \times m_{2}$ matrix functions $v(x, t)$, which are continuously differentiable and are such that $v_{x x}$ exists on the semi-strip $\mathcal{D}$. Moreover, we require that for each $k$ there is a value $\varepsilon_{k}=\varepsilon_{k}(v)>0$ such that $v$ is $k$ times continuously differentiable with respect to $x$ in the square

$$
\mathcal{D}\left(\varepsilon_{k}\right)=\left\{(x, t): 0 \leq x \leq \varepsilon_{k}, 0 \leq t \leq \varepsilon_{k}\right\}, \quad \mathcal{D}\left(\varepsilon_{k}\right) \subset \mathcal{D} .
$$

The class of such functions $v(x, t)$ is denoted by $C_{\varepsilon}(\mathcal{D})$.

Without loss of generality, we assume that the values $\varepsilon_{k}$ in (3.1) monotonically decrease. 
Proposition 3.2. Assume that $v \in C_{\varepsilon}(\mathcal{D})$ satisfies the $d N L S$ equation (1.1) on $\mathcal{D}$. Then, for each integer $r \geq 0$ and values $0 \leq k \leq r$, the functions $\left(\frac{\partial^{k}}{\partial t^{k}} v\right)(x, 0)$ and $\left(\frac{\partial^{k}}{\partial t^{k}} v_{x}\right)(x, 0)$ may be uniquely recovered (on the interval $0 \leq x \leq \varepsilon_{4 r}$ ) from the initial condition

$$
v(x, 0)=\mathcal{V}(x) .
$$

Moreover, on the domain $\mathcal{D}\left(\varepsilon_{4(r+1)}\right)$ the functions $\left(\frac{\partial^{k+1}}{\partial t^{k+1}} v\right),\left(\frac{\partial^{k+1}}{\partial t^{k+1}} v\right)_{x}$ and $\left(\frac{\partial^{k+1}}{\partial t^{k+1}} v\right)_{x x}$ exist and are continuous, and the equalities

$$
\left(\frac{\partial^{k}}{\partial t^{k}} v\right)_{x t}=\left(\frac{\partial^{k}}{\partial t^{k}} v\right)_{t x}, \quad\left(\frac{\partial^{k}}{\partial t^{k}} v\right)_{x x t}=\left(\frac{\partial^{k}}{\partial t^{k}} v\right)_{t x x}
$$

hold, whereas both sides of these equalities are again continuous. For $0 \leq k \leq r$ and $0 \leq \ell \leq s$ the functions $\left(\frac{\partial^{\ell}}{\partial x^{\ell}} \frac{\partial^{k+1}}{\partial t^{k+1}} v\right)$ exist and are continuous in the domains $\mathcal{D}\left(\varepsilon_{s+4(r+1)}\right)$.

In order to prove this proposition, we need a stronger version of the well-known Clairaut's (or Schwarz's) theorem on mixed derivatives. We need this version for the closed square $\mathcal{D}(\varepsilon)$ (as in Proposition 3.2 from [47]), which statement easily follows from the proofs of the mixed derivatives theorem for open domains (see, e.g., [53]).

Proposition $3.3([47])$. If the functions $f, f_{t}$ and $f_{t x}$ exist and are continuous on $\mathcal{D}(\varepsilon)$ and the derivative $f_{x}(x, 0)$ exists for $0 \leq x \leq \varepsilon$, then $f_{x}$ and $f_{x t}$ exist on $\mathcal{D}(\varepsilon)$ and $f_{x t}=f_{t x}$.

Proof of Proposition 3.2. We prove Proposition 3.2 by induction. First, consider the case $r=0$. Clearly, $v(x, 0)$ and $v_{x}(x, 0)$ are given by the initial condition (3.2). Since the right-hand side of (1.1) is two times continuously differentiable with respect to $x$ in $\mathcal{D}\left(\varepsilon_{4}\right)$, we derive that $v_{t}, v_{t x}$ and $v_{t x x}$ exist and are continuous in $\mathcal{D}\left(\varepsilon_{4}\right)$ :

$$
\begin{aligned}
& 2 v_{t}=\mathrm{i}\left(v_{x x}-2 v v^{*} v\right), \quad 2 v_{t x}=\mathrm{i}\left(v_{x x x}-2 \frac{\partial}{\partial x}\left(v v^{*} v\right)\right), \\
& 2 v_{t x x}=\mathrm{i}\left(v_{x x x x}-2 \frac{\partial^{2}}{\partial x^{2}}\left(v v^{*} v\right)\right) .
\end{aligned}
$$

Moreover, putting $f=v$ we see that conditions of Proposition 3.3 are fulfilled and the first equality in (3.3) holds for $k=0$. Putting $f=v_{x}$ and taking into account that the first equality in (3.3) yields $v_{x t}=v_{t x}$ and $v_{x t x}=v_{t x x}$, we see that conditions of Proposition 3.3 hold also for $f=v_{x}$. That is, $v_{x x t}$ exists and equals $v_{x t x}=v_{t x x}$. Thus, (3.3) is proved for $k=0$ (i.e., for $r=0)$. In view of (1.1), it is immediate that the last statement of Proposition 3.2 is also valid for $r=0$.

Next, assuming that the statements of Proposition 3.2 hold for all $0 \leq r \leq r_{0}$, let us prove them for $r=r_{0}+1$. Differentiating both sides of (1.1) $r_{0}$ times with respect to $t$ and taking into account (for $r_{0}>0$ and $k \leq r_{0}-1$ ) the second equality in (3.3), we express $\left(\frac{\partial^{r_{0}+1}}{\partial t^{r_{0}+1}} v\right)(x, 0)$ via derivatives which we already know. Then, from the first equality in (3.3), we obtain the formula $\left(\frac{\partial^{r_{0}+1}}{\partial t^{r_{0}+1}} v_{x}\right)(x, 0)=\left(\frac{\partial^{r_{0}+1}}{\partial t^{r_{0}+1}} v\right)_{x}(x, 0)$ and an expression for $\left(\frac{\partial^{r_{0}+1}}{\partial t^{r_{0}+1}} v_{x}\right)(x, 0)$ follows.

Differentiating both sides of (1.1) $r_{0}+1$ times and using (3.3) for $r=r_{0}$, we see also that the derivative $\frac{\partial^{r_{0}+2}}{\partial t^{r_{0}+2}} v$ exists and is continuous. Furthermore, differentiating (1.1) $r_{0}+1$ times with respect to $t$ and once or twice with respect to $x$, from the last statement of our proposition (for the case $\left.r=r_{0}\right)$ we derive that the derivatives $\left(\frac{\partial^{r_{0}+2}}{\partial t^{r_{0}+2}} v\right)_{x}$ and $\left(\frac{\partial^{r_{0}+2}}{\partial t^{r_{0}+2}} v\right)_{x x}$ exist and are continuous in $\mathcal{D}\left(\varepsilon_{4\left(r_{0}+2\right)}\right)$. Now, we see that the conditions of Proposition 3.3 are fulfilled for $f=\frac{\partial^{r_{0}+1}}{\partial t^{r_{0}+1}} v$, and so the first equality in (3.3) holds for $k \leq r_{0}+1$. Using this first equality 
in (3.3), we derive that the conditions of Proposition 3.3 are fulfilled for $f=\left(\frac{\partial^{r_{0}+1}}{\partial t^{r_{0}+1}} v\right)_{x}$ and therefore the second equality in (3.3) holds for $k \leq r_{0}+1$. Differentiating again both sides of (1.1), we show that the last statement in Proposition 3.2 holds for $r=r_{0}+1$.

The class $C\left(\left\{\widetilde{M}_{k}\right\}\right)$ consists of all infinitely differentiable on $[0, a)$ scalar functions $f$ such that for some $c(f) \geq 0$ and for fixed constants $\widetilde{M}_{k}>0(k \geq 0)$ we have

$$
\left|\frac{d^{k} f}{d x^{k}}(x)\right| \leq c(f)^{k+1} \widetilde{M}_{k} \quad \text { for all } \quad x \in[0, a) .
$$

Here, we use the notation $\widetilde{M}_{k}$ (as well $\widetilde{M}$ below) because the upper estimates $M$ (without tilde) were already used in Section 2. Recall that $C\left(\left\{\widetilde{M}_{k}\right\}\right)$ is called quasi-analytic if for the functions $f$ from this class and for any $0 \leq x<a$ the equalities $\frac{d^{k} f}{d x^{k}}(x)=0(k \geq 0)$ yield $f \equiv 0$. According to the famous Denjoy-Carleman theorem, the equality

$$
\sum_{n=1}^{\infty} \frac{1}{L_{n}}=\infty, \quad L_{n}:=\inf _{k \geq n} \widetilde{M}_{k}^{1 / k}
$$

implies that the class $C\left(\left\{\widetilde{M}_{k}\right\}\right)$ is quasi-analytic.

Corollary 3.4. If $v(x, t)$ satisfies conditions of Proposition 3.2 and the entries of $v(0, t)$ or $v_{x}(0, t)$ are quasi-analytic, then the matrix functions $v(0, t)$ or $v_{x}(0, t)$, respectively, are uniquely defined by the initial condition (3.2).

Let us consider the case, where both matrix functions $v(0, t)$ and $v_{x}(0, t)$ are quasi-analytic. More precisely, we assume that the entries $v_{i j}(0, t)$ of $v(0, t)$ belong to some quasi-analytic classes $C\left(\left\{\widetilde{M}_{k}(i, j)\right\}\right)$, the entries $\left(v_{i j}\right)_{x}(0, t)$ of $v_{x}(0, t)$ belong to some quasi-analytic classes $C\left(\left\{\widetilde{M}_{k}^{+}(i, j)\right\}\right)$, and, in this case, we say that $v(0, t) \in C([0, a) ; \widetilde{M})$ and $v_{x}(0, t) \in C\left([0, a) ; \widetilde{M}^{+}\right)$, where

$$
\widetilde{M}=\left\{\widetilde{M}_{k}(i, j)\right\} \quad \text { and } \quad \widetilde{M}^{+}=\left\{\widetilde{M}_{k}^{+}(i, j)\right\} .
$$

Now, using Proposition 2.5, Theorem 2.7, Remark 2.8 and Corollary 3.4 we obtain the main theorem in this section.

Theorem 3.5. Assume that $v \in C_{\varepsilon}(\mathcal{D})$ satisfies the $d N L S$ equation (1.1) on $\mathcal{D}$, that (2.8) holds and that boundary values $v(0, t)$ and $v_{x}(0, t)$ belong to quasi-analytic classes $C([0, a) ; \widetilde{M})$ and $C\left([0, a) ; \widetilde{M}^{+}\right)$, respectively. Then, $v$ is uniquely defined by the initial condition (3.2).

Remark 3.6. We see that the scheme to recover $v$ (in the semi-strip $\mathcal{D}$ ) from the initial condition follows from Proposition 2.5, Theorem 2.7 and the proof of Proposition 3.2. The only step that we did not describe in detail is the recovery of the functions $v(0, t)$ and $v_{x}(0, t)$ from their Taylor coefficients at $t=0$. Although Taylor coefficients uniquely determine quasi-analytic functions $v(0, t)$ and $v_{x}(0, t)$, the recovery of these functions presents an interesting problem, which is not solved completely so far. See [4, 34] and [8, Section III.8] for some important results.

Another important case, where the boundary conditions of the nonlinear Schrödinger equation determine a quasi-analytic initial condition, is discussed in [47, Section 3]. We note that our solutions are not (in general) quasi-analytic. 
Remark 3.7. An interesting class of such solutions of a scalar dNLS that the Weyl functions

$\widetilde{\varphi}(t, z)$ may be presented as the series $\widetilde{\varphi}(t, z)=\sum_{k=0}^{\infty} \alpha_{k}(t) / z^{k}$ (for sufficiently large values of $z$ ) was treated in [50]. (We note that the Weyl functions $\widetilde{\varphi}(z)$ from [50] are Herglotz functions and can be easily mapped into the Weyl functions $\varphi(z)$ considered here via a linear-fractional transformation with constant coefficients.) According to [50, Theorem 1], if $\widetilde{\varphi}(0, z)=\sum_{k=0}^{\infty} \alpha_{k}(0) / z^{k}$, there is one and only one solution of dNLS from this class in some semi-strip.

Important results on the asymptotics of Weyl functions are given, for instance, in $[14,26]$. However, it would be fruitful to know, under which conditions the asymptotic series (for Weyl functions) from $[14,26]$ converge or at least uniquely define the corresponding Weyl function.

\section{Nonlinear optics equation on a semi-strip}

In this section we consider the nonlinear optics equation (1.8), where $D$ has the form (1.9). We consider equation (1.8) on the semi-strip $\mathcal{D}$, which is given by (1.6). First, using Weyl theoretic results from Section 2.2, we express Weyl function $\varphi(t, z)$ of the auxiliary system $(2.10)$, where $\zeta(x)=\zeta(x, t)=[D, \varrho(x, t)]$, in terms of $\varphi(0, z)$ and the boundary condition $\varrho(0, t)=\widehat{\rho}(t)$. In other words, we express in these terms the evolution of the Weyl function. For that purpose, following formulas (2.5) and (2.6) in Proposition 2.6, we introduce matrix function $R(t, z)$ by the relations

$$
R_{t}(t, z)=(\mathrm{i} z \widehat{D}-[\widehat{D}, \widehat{\rho}(t)]) R(t, z), \quad R(0, z)=I_{m} .
$$

Recall that $\widehat{D}$ is a diagonal matrix and that $\widehat{D}>0$.

Theorem 4.1. Let $\varrho(x, t)$ satisfy the nonlinear optics equation (1.8) and the boundary condition $\varrho(0, t)=\widehat{\rho}(t)$. Assume that $\varrho$ is uniformly bounded and continuously differentiable on $\mathcal{D}$. Then, the matrix functions

$$
\breve{\psi}_{k}(t, z):=\left[\begin{array}{ll}
I_{k} & 0
\end{array}\right] R(t, z) \varphi(0, z)\left[\begin{array}{c}
0 \\
I_{m-k}
\end{array}\right]\left(\left[\begin{array}{ll}
0 & I_{m-k}
\end{array}\right] R(t, z) \varphi(0, z)\left[\begin{array}{c}
0 \\
I_{m-k}
\end{array}\right]\right)^{-1}
$$

are well-defined for $1 \leq k<m$, and the evolution of the Weyl function is given by the formula

$$
\left\{\varphi_{i, k+1}(t, z)\right\}_{i=1}^{k}=\breve{\psi}_{k}(t, z)\left[\begin{array}{c}
1 \\
0 \\
\ldots \\
0
\end{array}\right], \quad \Im(z)<-M
$$

and by the normalization conditions (2.18).

Proof. We set

$$
M_{0}=\sup \|\zeta(x, t)\|, \quad(x, t) \in \mathcal{D}, \quad M>\max _{1 \leq k<m}\left(2 M_{0} /\left(d_{k}-d_{k+1}\right)\right) .
$$

Recall that $G$ and $F$ for the case of the nonlinear optics equation are given by (2.2). Since $\varrho$ is continuously differentiable, the conditions of Proposition 2.6 are fulfilled. Taking into account that the fundamental solution of (2.10) is denoted by $w$ (instead of $u$ in Proposition 2.6), we rewrite $(2.7)$ in the form $w(x, t, z)=R(x, t, z) w(x, 0, z) R(t, z)^{-1}$ or, equivalently,

$$
w(x, t, z)^{-1}=R(t, z) w(x, 0, z)^{-1} R(x, t, z)^{-1} .
$$


Using (4.5), we express $w(x, t, z)^{-1}$ via $w(x, \widetilde{t}, z)^{-1}, 0 \leq t, \widetilde{t}<a$ :

$$
\begin{aligned}
w(x, t, z)^{-1} & =R(t, z) R(\widetilde{t}, z)^{-1} R(\widetilde{t}, z) w(x, 0, z)^{-1} R(x, \widetilde{t}, z)^{-1}\left(R(x, t, z) R(x, \widetilde{t}, z)^{-1}\right)^{-1} \\
& =R(t, z) R(\widetilde{t}, z)^{-1} w(x, \widetilde{t}, z)^{-1}\left(R(x, t, z) R(x, \widetilde{t}, z)^{-1}\right)^{-1} .
\end{aligned}
$$

Recall that $R_{t}=F R$, where $F$ is given in $(2.2)$ and $\widehat{D}>0$. Hence, putting $\mathcal{R}(x, t, \widetilde{t}, z):=$ $\left(R(x, t, z) R(x, \widetilde{t}, z)^{-1}\right)^{-1}$ we derive

$$
\begin{aligned}
& \frac{\partial}{\partial t} \mathcal{R}(x, t, \widetilde{t}, z)=-\mathcal{R}(x, t, \widetilde{t}, z) F(x, t, z), \\
& \frac{\partial}{\partial t}\left(\mathcal{R}(x, t, \widetilde{t}, z) \mathcal{R}(x, t, \widetilde{t}, z)^{*}\right)<0, \quad \Im(z)<0, \quad \mathcal{R}(x, \widetilde{t}, \widetilde{t}, z)=I_{m} .
\end{aligned}
$$

From the relations above it is immediate that

$$
\|\mathcal{R}(x, t, \tilde{t}, z)\|<1 \quad \text { for } \quad t>\widetilde{t}, \quad \Im(z)<0,
$$

which allows us to estimate the difference

$$
I_{m}-\mathcal{R}(x, t, \widetilde{t}, z)=\int_{\widetilde{t}}^{t} \mathcal{R}(x, s, \widetilde{t}, z) F(x, s, z) d s .
$$

According to (4.4), (4.7) and (4.8), for each $\delta>0$ and $c>M$ there is $\varepsilon=\varepsilon\left(M_{0}\right)>0$ such that

$$
\begin{aligned}
& \left\|I_{m}-\mathcal{R}(x, t, \widetilde{t}, z)\right\| \leq \delta \\
& \text { for all } \quad x \in[0, \infty), \quad 0 \leq t-\widetilde{t} \leq \varepsilon, \quad z \in\{z:|z|<c\} \cap\{z: \Im(z)<-M\}, \quad c>M . \\
& \text { ifying } \left.(2.20) \text { (so that the functions } \psi_{k} \text { and } w \text { depend there also on an additional variable } t\right), \\
& \begin{aligned}
\psi_{k}(x, t, z)= & {\left[\begin{array}{ll}
I_{k} & 0
\end{array}\right] R(t, z) R(\widetilde{t}, z)^{-1} w(x, \widetilde{t}, z)^{-1} \mathcal{R}(x, t, \widetilde{t}, z) \mathcal{Q}_{k}(z) } \\
& \times\left(\left[\begin{array}{ll}
0 & I_{m-k}
\end{array}\right] R(t, z) R(\widetilde{t}, z)^{-1} w(x, \widetilde{t}, z)^{-1} \mathcal{R}(x, t, \widetilde{t}, z) \mathcal{Q}_{k}(z)\right)^{-1} .
\end{aligned}
\end{aligned}
$$

Modifying (2.20) (so that the functions $\psi_{k}$ and $w$ depend there also on an additional variable $t$ ), in view of (4.6), we derive

Moreover, putting

$$
\widetilde{\mathcal{Q}}_{k}(z):=\mathcal{R}(x, t, \widetilde{t}, z) \mathcal{Q}_{k}(z), \quad \mathcal{Q}_{k}(z):=\left[\begin{array}{ll}
0 & I_{m-k}
\end{array}\right]
$$

we see that for sufficiently small $\delta$ the matrix function $\widetilde{\mathcal{Q}}_{k}(z)$ satisfies $(2.19)$ in the domain (for $z$ ) given in (4.9). Substituting $\widetilde{\mathcal{Q}}_{k}$ (instead of $\mathcal{Q}_{k}$ ) into $(2.20)$, we obtain

$$
\begin{aligned}
& {\left[\begin{array}{c}
\psi_{k}(x, \widetilde{t}, z) \\
I_{m-k}
\end{array}\right]=w(x, \widetilde{t}, z)^{-1} \widetilde{\mathcal{Q}}_{k}(z)\left(\left[\begin{array}{ll}
0 & I_{m-k}
\end{array}\right] w(x, \widetilde{t}, z)^{-1} \widetilde{\mathcal{Q}}_{k}(z)\right)^{-1}, \quad \text { i.e. },} \\
& w(x, \widetilde{t}, z)^{-1} \widetilde{\mathcal{Q}}_{k}(z)=\left[\begin{array}{c}
\psi_{k}(x, \widetilde{t}, z) \\
I_{m-k}
\end{array}\right]\left(\left[\begin{array}{ll}
0 & I_{m-k}
\end{array}\right] w(x, \widetilde{t}, z)^{-1} \widetilde{\mathcal{Q}}_{k}(z)\right) .
\end{aligned}
$$

Using the first equality in (4.11), we can substitute (4.12) into (4.10). Thus, we derive

$$
\left[\begin{array}{c}
\psi_{k}(x, t, z) \\
I_{m-k}
\end{array}\right]=R(t, z) R(\widetilde{t}, z)^{-1}\left[\begin{array}{c}
\psi_{k}(x, \widetilde{t}, z) \\
I_{m-k}
\end{array}\right]\left(\left[\begin{array}{ll}
0 & I_{m-k}
\end{array}\right] R(t, z) R(\widetilde{t}, z)^{-1}\left[\begin{array}{c}
\psi_{k}(x, \widetilde{t}, z) \\
I_{m-k}
\end{array}\right]\right)^{-1}
$$

and in view of (2.22), passing to the limit $x \rightarrow \infty$, we have the following formula

$$
\begin{aligned}
{\left[\begin{array}{c}
\breve{\psi}_{k}(t, z) \\
I_{m-k}
\end{array}\right]=} & R(t, z) R(\widetilde{t}, z)^{-1}\left[\begin{array}{c}
\breve{\psi}_{k}(\widetilde{t}, z) \\
I_{m-k}
\end{array}\right] \\
& \times\left(\left[\begin{array}{ll}
0 & I_{m-k}
\end{array}\right] R(t, z) R(\widetilde{t}, z)^{-1}\left[\begin{array}{c}
\breve{\psi}_{k}(\widetilde{t}, z) \\
I_{m-k}
\end{array}\right]\right)^{-1}
\end{aligned}
$$


Here we used the fact that, according to (2.21) and (4.9),

$$
\operatorname{det}\left(\left[\begin{array}{ll}
0 & I_{m-k}
\end{array}\right] R(t, z) R(\widetilde{t}, z)^{-1}\left[\begin{array}{c}
\breve{\psi}_{k}(\widetilde{t}, z) \\
I_{m-k}
\end{array}\right]\right) \neq 0
$$

for sufficiently small $\delta$. Setting $\widetilde{t}=k \varepsilon(k=0,1, \ldots)$, recalling that $R(0, z)=I_{m}$ and applying each time (4.13), we easily prove (by induction) the equality

$$
\left[\begin{array}{c}
\breve{\psi}_{k}(t, z) \\
I_{m-k}
\end{array}\right]=R(t, z)\left[\begin{array}{c}
\breve{\psi}_{k}(0, z) \\
I_{m-k}
\end{array}\right]\left(\left[\begin{array}{ll}
0 & I_{m-k}
\end{array}\right] R(t, z)\left[\begin{array}{c}
\breve{\psi}_{k}(0, z) \\
I_{m-k}
\end{array}\right]\right)^{-1}
$$

for $t$ on all intervals $[0,(k+1) \varepsilon] \cap[0, a)$, that is, for $t$ on $[0, a)$. Although (4.14) is proved for $z \in\{z:|z|<c\} \cap\{z: \Im(z)<-M\}$, the analyticity of both sides of (4.14) implies that the equality holds in the semi-plane $\Im(z)<-M$. Finally, we note that $(2.24)$ at $t=0$ yields

$$
\left[\begin{array}{c}
\breve{\psi}_{k}(0, z) \\
I_{m-k}
\end{array}\right]=\varphi(0, z)\left[\begin{array}{c}
0 \\
I_{m-k}
\end{array}\right]\left(\left[\begin{array}{ll}
0 & I_{m-k}
\end{array}\right] \varphi(0, z)\left[\begin{array}{c}
0 \\
I_{m-k}
\end{array}\right]\right)^{-1}
$$

Substituting (4.15) into (4.14), we obtain (4.2). The procedure to recover $\varphi(t, z)$ from $\left\{\breve{\psi}_{k}(t, z)\right\}$ (for fixed values of $t$ ) is described in Section 2.2 (note that (4.3) coincides with (2.23)).

Now, let us prove a uniqueness result for the case of $\widehat{D}$ of the form (1.10). Let initial condition be given by the equality

$$
\varrho(x, 0)=\rho(x), \quad \sup _{x \in[0, \infty)}\|\rho(x)\|<\infty .
$$

Denote the Weyl function of system

$$
y_{x}(x, z)=(\mathrm{i} z D-\zeta(x)) y(x, z), \quad x \geq 0, \quad \zeta=[D, \rho]
$$

by $\varphi_{0}(z)$. (According to Section 2.2, this Weyl function exists and is unique.)

Theorem 4.2. For the case where the entries of the matrix $\widehat{D}$ in (1.8) are ordered as in (1.10), there is no more than one uniformly bounded and continuously differentiable on $\mathcal{D}$ solution $\varrho=\varrho^{*}$ (of the nonlinear optics equation (1.8)), having the initial values $\varrho(x, 0)$ such that $\varphi_{0}$ is bounded and (2.13) holds. That is, there is no more than one solution of the corresponding initial value problem.

Proof. Let $\varrho(x, t)$ satisfy conditions of the theorem. We fix $M$ such (4.4) is valid, $\varphi_{0}(z)$ is bounded for $\Im(z) \leq-M$ and (2.13) holds for $\varphi_{0}(\xi+\mathrm{i} \eta)$ when $\eta \leq-M$. We set also

$$
\widehat{M}=\sup _{0 \leq t<a}\|\widehat{\zeta}(0, t)\|, \quad \widehat{\zeta}(0, t)=[\widehat{D}, \widehat{\rho}(t)]
$$

where $\widehat{\rho}(t)=\varrho(0, t)$. First, we show that the inequality

$$
\sup _{t \in[0, a), \Im(z)<-M}\left\|R(t, z) \varphi_{0}(z) \exp \{-\mathrm{i} z t \widehat{D}\}\right\|<\infty, \quad a<\infty
$$

is valid. Indeed, according to (2.21) and (4.14) we have

$$
\left[\breve{\psi}_{k}(0, z)^{*} \quad I_{m-k}\right] R(t, z)^{*} J_{k} R(t, z)\left[\begin{array}{c}
\breve{\psi}_{k}(0, z) \\
I_{m-k}
\end{array}\right] \leq 0
$$


Clearly, (4.19) yields the inequality

$$
\begin{gathered}
-4 \widehat{M}\left[\breve{\psi}_{k}(0, z)^{*} \quad I_{m-k}\right] R(t, z)^{*}\left(I_{m}-J_{k}\right) R(t, z)\left[\begin{array}{c}
\breve{\psi}_{k}(0, z) \\
I_{m-k}
\end{array}\right] \\
\leq-4 \widehat{M}\left[\breve{\psi}_{k}(0, z)^{*} \quad I_{m-k}\right] R(t, z)^{*} R(t, z)\left[\begin{array}{c}
\breve{\psi}_{k}(0, z) \\
I_{m-k}
\end{array}\right] .
\end{gathered}
$$

Taking into account that $R_{t}=F R$ and relations (1.10), (2.2), (4.17) and (4.20) hold, we derive

$$
\begin{array}{r}
\frac{d}{d t}\left(\exp \left\{\mathrm{i}(\bar{z}-z) \widehat{d}_{k+1} t-4 \widehat{M} t\right\}\left[\breve{\psi}_{k}(0, z)^{*} \quad I_{m-k}\right]\right. \\
\left.\quad \times R(t, z)^{*}\left(I_{m}-J_{k}\right) R(t, z)\left[\begin{array}{c}
\breve{\psi}_{k}(0, z) \\
I_{m-k}
\end{array}\right]\right) \leq 0
\end{array}
$$

for $\Im(z)<0$. Formulas (4.19) and (4.21) imply that

$$
\begin{aligned}
& \exp \left\{\mathrm{i}(\bar{z}-z) \widehat{d}_{k+1} t-4 \widehat{M} t\right\}\left[\breve{\psi}_{k}(0, z)^{*} \quad I_{m-k}\right] R(t, z)^{*} R(t, z)\left[\begin{array}{c}
\breve{\psi}_{k}(0, z) \\
I_{m-k}
\end{array}\right] \\
& \quad \leq\left[\breve{\psi}_{k}(0, z)^{*} \quad I_{m-k}\right]\left(I_{m}-J_{k}\right)\left[\begin{array}{c}
\breve{\psi}_{k}(0, z) \\
I_{m-k}
\end{array}\right]=2 I_{m-k}, \quad 1 \leq k<m, \quad \Im(z)<-M
\end{aligned}
$$

Recall that $\varphi_{0}$ is given by (2.23). Hence, (4.18) follows from (4.22).

Consider system (4.1). Since $\varphi_{0}$ is bounded and satisfies (2.13) and (4.18), according to Corollary 2.13, the matrix function $\widehat{\zeta}(0, t)=[\widehat{D}, \widehat{\rho}(t)]$ (and so $R$ ) is uniquely defined by $\varphi_{0}$. Thus, from Theorem 4.1, we see that $\varphi(t, z)$ is uniquely defined by $\varphi_{0}$.

In order to prove our theorem, it remains to show that $\varphi(t, z)$ satisfies conditions of Theorem 2.12 for each $t$. Indeed, in view of (4.1), we can rewrite for $R$ the representation (2.25):

$$
R(t, z)=\exp \{\mathrm{i} z t \widehat{D}\}+\int_{\widehat{d}_{m} t}^{\widehat{d}_{1} t} \exp \{\mathrm{i} z s\} \widehat{N}(t, s) d s, \quad \sup _{t<a}\|\widehat{N}(t, s)\|<\infty .
$$

By virtue of (4.18), the matrix function $R(t, z) \varphi_{0}(z) \mathrm{e}^{-\mathrm{i} z t \widehat{D}}-I_{m}$ is bounded in the domain $\Im(z) \leq-M$. Since $R(t, z)$ satisfies (4.23) and $M$ is chosen so that $\varphi_{0}$ satisfies (2.13) for $z=\xi-\mathrm{i} M$, we see that $R(t, \xi-\mathrm{i} M) \varphi_{0}(\xi-\mathrm{i} M) \mathrm{e}^{-\mathrm{i}(\xi-\mathrm{i} M) t \widehat{D}}-I_{m} \in L_{m \times m}^{2}(-\infty, \infty)$ for each $0 \leq t<a$, where $L_{m \times m}^{2}(0, \infty)$ is the class of $m \times m$ matrix functions, the entries of which belong to $L^{2}(0, \infty)$. Hence, the well-known Theorems V and VIII (Sections 4 and 5 in [38], respectively) on the Fourier transform in complex domains yield the representation (see [48, formula (E11)]):

$$
R(t, z) \varphi_{0}(z) \mathrm{e}^{-\mathrm{i} z t \widehat{D}}=I_{m}+\int_{0}^{\infty} \mathrm{e}^{-\mathrm{i} z x} \mathcal{F}(x) d x, q \quad \mathrm{e}^{-x M} \mathcal{F}(x) \in L_{m \times m}^{2}(0, \infty) .
$$

It is immediate also that formula (4.2) can be modified slightly:

$$
\begin{aligned}
\breve{\psi}_{k}(t, z)= & {\left[\begin{array}{ll}
I_{k} & 0
\end{array}\right] R(t, z) \varphi_{0}(z) \mathrm{e}^{-\mathrm{i} z t \widehat{D}}\left[\begin{array}{c}
0 \\
I_{m-k}
\end{array}\right] } \\
& \times\left(\left[\begin{array}{ll}
0 & I_{m-k}
\end{array}\right] R(t, z) \varphi_{0}(z) \mathrm{e}^{-\mathrm{i} z t \widehat{D}}\left[\begin{array}{c}
0 \\
I_{m-k}
\end{array}\right]\right)^{-1} .
\end{aligned}
$$

According to (4.24), the normalized GW-function $\varphi(t, z)$ constructed via equalities (4.3) and (4.25) satisfies conditions of Theorem 2.12. In other words, there is no more then one solution of ISpP for $\varphi(t, z)$, that is, $\varrho(x, t)$ is unique. 
Remark 4.3. In the case of system (4.1), by virtue of (4.17) and (4.18), the requirements of Corollary 2.13 are fulfilled for $\varphi_{0}$, and so $\varphi_{0}$ uniquely determines the boundary condition $\widehat{\rho}$. Moreover, if $\varphi_{0}$ satisfies (2.14) and (2.15) there is a rigorous procedure to recover $\widehat{\rho}$ from $\varphi_{0}$ (see Remark 2.14).

Although Theorem 4.2 was announced in [41], its proof is published for the first time. It is essential to know, for which initial conditions $\rho(x)$, the restrictions on $\varphi_{0}$ (from Theorem 4.2) are fulfilled. First, let us formulate a particular case of Theorem 6.1 from [5].

Proposition 4.4. Suppose that the $m \times m$ matrix function $\rho(x)$ is absolutely continuous on $\mathbb{R}$ and $\rho(x), \rho^{\prime}(x) \in L_{m \times m}^{1}(-\infty, \infty)$. Then, for some $M>0$, there is an analytic with respect to $z$ fundamental (unnormalized) solution $\mathcal{M}(x, z)$ of the equation

$$
\mathcal{M}_{x}=\mathrm{i} z[D, \mathcal{M}]-\zeta \mathcal{M}, \quad x \in \mathbb{R}, \quad \zeta=[D, \rho],
$$

such that uniformly with respect to $x$ we have

$$
\mathcal{M}(x, z)=I_{m}+\frac{1}{z} \mathcal{M}_{1}(x)+o\left(|z|^{-1}\right), \quad|z| \rightarrow \infty, \quad \Im z<-M,
$$

where $\mathcal{M}_{1}(x)$ is absolutely continuous.

We note that the fact that $\mathcal{M}_{1}$ is absolutely continuous is immediate from the proof of [5, Theorem 6.1] (more precisely, from formulas (6.6) and (6.8)). Now, since we can always extend $\rho^{\prime}$ on $\mathbb{R}$, we consider $\rho(x)$ on $[0, \infty)$ only and assume that $\rho$ is absolutely continuous and $\rho, \rho^{\prime} \in L_{m \times m}^{1}\left(\mathbb{R}_{+}\right)$. Without loss of generality, we assume that $M$ is chosen so that $\mathcal{M}(0, z)$ is invertible for $\Im(z)<-M$. Then, according to (4.26), the matrix function

$$
w(x, z)=\mathcal{M}(x, z) \mathrm{e}^{\mathrm{i} z x D} \mathcal{M}(0, z)^{-1}
$$

is the normalized (by $w(0, z)=I_{m}$ ) fundamental solution of the equation (4.16) (and we don't require so far that $\left.\rho=\rho^{*}\right)$. Moreover, (4.27) implies that (2.12) holds for $\varphi(z)=\mathcal{M}(0, z)$. That is, assuming $\rho=\rho^{*}$ and taking into account Definition 2.9, we see that $\mathcal{M}(0, z)$ is a GW-function of (4.16).

Recall that in Theorem 4.2 we speak about the Weyl function $\varphi_{0}$ or, equivalently for a bounded function $\rho=\rho^{*}$, about the normalized GW-function. Thus, we should normalize $\mathcal{M}(0, z)$. For that purpose we construct a lower triangular matrix function $\widehat{\mathcal{M}}(z)$ via the right lower $k \times k$ blocks $\mathcal{P}_{k}(z)$ of $\mathcal{M}(0, z)$. Namely, we construct $\widehat{\mathcal{M}}(z)$ columnwise via the equalities

$$
\widehat{\mathcal{M}}(z)\left\{\delta_{i, m-k+1}\right\}_{i=1}^{m}:=\left[\begin{array}{c}
0 \\
\mathcal{P}_{k}(z)^{-1}\left\{\delta_{i 1}\right\}_{i=1}^{k}
\end{array}\right], \quad 1 \leq k \leq m,
$$

where $\left\{\delta_{i, m-k+1}\right\}_{i=1}^{m}$ and $\left\{\delta_{i 1}\right\}_{i=1}^{k}$ are column vectors. It follows from (4.29) that the normalization conditions (2.18) hold for

$$
\varphi_{0}(z)=\mathcal{M}(0, z) \widehat{\mathcal{M}}(z) .
$$

Since $\mathcal{M}(z)$ is lower triangular and $D$ satisfies (1.9), we see that $\mathrm{e}^{\mathrm{i} z x D} \widehat{\mathcal{M}}(z) \mathrm{e}^{-\mathrm{i} z x D}$ is bounded for $\Im(z)<-M$. Hence, taking into account that $\mathcal{M}(0, z)$ is a $\mathrm{GW}$-function, we derive that (2.12) is also valid for $\varphi(z)=\mathcal{M}(0, z) \widehat{\mathcal{M}}(z)$ (i.e., $\varphi_{0}$ given by (4.30) is the normalized GW-function). Finally, relations (4.27), (4.29) and (4.30) show that $\varphi_{0}$ is bounded and that (2.13) also holds for $\varphi_{0}$. Thus, we proved the statement below.

Proposition 4.5. Suppose that the initial condition $\rho(x)=\rho(x)^{*}$ is absolutely continuous on $[0, \infty)$ and $\rho(x), \rho^{\prime}(x) \in L_{m \times m}^{1}\left(\mathbb{R}_{+}\right)$. Then, the Weyl function $\varphi_{0}(z)$ of the system (4.16), where $\zeta=\zeta_{0}=[D, \rho]$, exists. Moreover, $\varphi_{0}(z)$ is analytic and bounded (in some semi-plane $\Im(z)<-M, M>0$ ), and it satisfies (2.13).

An existence result for a solution of an initial value problem (for the nonlinear optics equation) is given in [44, Remark 4.7]. 


\section{Acknowledgements}

This research was supported by the Austrian Science Fund (FWF) under Grant No. P24301. The author is grateful to A. Rainer for a helpful discussion on quasi-analytic functions.

\section{References}

[1] Ablowitz M.J., Haberman R., Resonantly coupled nonlinear evolution equations, J. Math. Phys. 16 (1975), $2301-2305$.

[2] Ablowitz M.J., Prinari B., Trubatch A.D., Discrete and continuous nonlinear Schrödinger systems, London Mathematical Society Lecture Note Series, Vol. 302, Cambridge University Press, Cambridge, 2004.

[3] Ashton A.C.L., On the rigorous foundations of the Fokas method for linear elliptic partial differential equations, Proc. R. Soc. Lond. Ser. A Math. Phys. Eng. Sci. 468 (2012), 1325-1331.

[4] Bang T., On quasi-analytic functions, in C. R. Dixième Congrès Math. Scandinaves 1946, Jul. Gjellerups Forlag, Copenhagen, 1947, 249-254.

[5] Beals R., Coifman R.R., Scattering and inverse scattering for first order systems, Comm. Pure Appl. Math. 37 (1984), 39-90.

[6] Berezanskii Yu.M., Integration of non-linear difference equations by means of inverse problem technique, Dokl. Akad. Nauk SSSR 281 (1985), 16-19.

[7] Berezanskii Yu.M., Gekhtman M.I., Inverse problem of spectral analysis and nonabelian chains of nonlinear equations, Ukrain. Math. J. 42 (1990), 645-658.

[8] Beurling A., The collected works of Arne Beurling. Vol. 1. Complex analysis, Contemporary Mathematicians, Birkhäuser Boston, Inc., Boston, MA, 1989.

[9] Bikbaev R.F., Tarasov V.O., Initial-boundary value problem for the nonlinear Schrödinger equation, J. Phys. A: Math. Gen. 24 (1991), 2507-2516.

[10] Bona J., Winther R., The Korteweg-de Vries equation, posed in a quarter-plane, SIAM J. Math. Anal. 14 (1983), 1056-1106.

[11] Bona J.L., Fokas A.S., Initial-boundary-value problems for linear and integrable nonlinear dispersive partial differential equations, Nonlinearity 21 (2008), T195-T203.

[12] Carroll R., Bu Q., Solution of the forced nonlinear Schrödinger (NLS) equation using PDE techniques, Appl. Anal. 41 (1991), 33-51.

[13] Chu C.K., Xiang L.W., Baransky Y., Solitary waves induced by boundary motion, Comm. Pure Appl. Math. 36 (1983), 495-504.

[14] Clark S., Gesztesy F., Weyl-Titchmarsh $M$-function asymptotics, local uniqueness results, trace formulas, and Borg-type theorems for Dirac operators, Trans. Amer. Math. Soc. 354 (2002), 3475-3534, math.SP/0102040.

[15] Damanik D., Killip R., Simon B., Perturbations of orthogonal polynomials with periodic recursion coefficients, Ann. of Math. 171 (2010), 1931-2010, math.SP/0702388.

[16] Degasperis A., Manakov S.V., Santini P.M., Mixed problems for linear and soliton partial differential equations, Theoret. and Math. Phys. 133 (2002), 1475-1489.

[17] Fokas A.S., Integrable nonlinear evolution equations on the half-line, Comm. Math. Phys. 230 (2002), 1-39.

[18] Fokas A.S., A unified approach to boundary value problems, CBMS-NSF Regional Conference Series in Applied Mathematics, Vol. 78, Society for Industrial and Applied Mathematics (SIAM), Philadelphia, PA, 2008.

[19] Fokas A.S., Pelloni B. (Editors), Unified transform for boundary value problems. Applications and advances, Society for Industrial and Applied Mathematics (SIAM), Philadelphia, PA, 2015.

[20] Fritzsche B., Kirstein B., Roitberg I.Ya., Sakhnovich A.L., Weyl theory and explicit solutions of direct and inverse problems for Dirac system with a rectangular matrix potential, Oper. Matrices 7 (2013), 183-196, arXiv:1105.2013.

[21] Fritzsche B., Kirstein B., Sakhnovich A.L., Weyl functions of generalized Dirac systems: integral representation, the inverse problem and discrete interpolation, J. Anal. Math. 116 (2012), 17-51, arXiv:1007.4304. 
[22] Gesztesy F., Mitrea M., Zinchenko M., On Dirichlet-to-Neumann maps and some applications to modified Fredholm determinants, in Methods of Spectral Analysis in Mathematical Physics, Oper. Theory Adv. Appl., Vol. 186, Birkhäuser Verlag, Basel, 2009, 191-215, arXiv:1002.0390.

[23] Gesztesy F., Weikard R., Zinchenko M., Initial value problems and Weyl-Titchmarsh theory for Schrödinger operators with operator-valued potentials, Oper. Matrices 7 (2013), 241-283, arXiv:1109.1613.

[24] Gohberg I., Kaashoek M.A., Sakhnovich A.L., Scattering problems for a canonical system with a pseudoexponential potential, Asymptot. Anal. 29 (2002), 1-38.

[25] Habibullin I.T., Backlund transformation and integrable boundary-initial value problems, in Nonlinear World, Vol. 1 (Kiev, 1989), World Sci. Publ., River Edge, NJ, 1990, 130-138.

[26] Harris B.J., The asymptotic form of the Titchmarsh-Weyl $m$-function associated with a Dirac system, J. London Math. Soc. 31 (1985), 321-330.

[27] Holmer J., The initial-boundary-value problem for the 1D nonlinear Schrödinger equation on the half-line, Differential Integral Equations 18 (2005), 647-668, math.AP/0602152.

[28] Kac M., van Moerbeke P., A complete solution of the periodic Toda problem, Proc. Nat. Acad. Sci. USA 72 (1975), 2879-2880.

[29] Kaikina E.I., Inhomogeneous Neumann initial-boundary value problem for the nonlinear Schrödinger equation, J. Differential Equations 255 (2013), 3338-3356.

[30] Kamvissis S., Shepelsky D., Zielinski L., Robin boundary condition and shock problem for the focusing nonlinear Schrödinger equation, J. Nonlinear Math. Phys. 22 (2015), 448-473, arXiv:1412.7636.

[31] Kaup D.J., The forced Toda lattice: an example of an almost integrable system, J. Math. Phys. 25 (1984), $277-281$.

[32] Kaup D.J., Newell A.C., The Goursat and Cauchy problems for the sine-Gordon equation, SIAM J. Appl. Math. 34 (1978), 37-54.

[33] Kaup D.J., Steudel H., Recent results on second harmonic generation, in Recent Developments in Integrable Systems and Riemann-Hilbert Problems (Birmingham, AL, 2000), Contemp. Math., Vol. 326, Amer. Math. Soc., Providence, RI, 2003, 33-48.

[34] Khryptun V.G., Expansion of functions of quasi-analytic classes in series in polynomials, Ukrain. Math. J. 41 (1989), 569-574.

[35] Kostenko A., Sakhnovich A., Teschl G., Weyl-Titchmarsh theory for Schrödinger operators with strongly singular potentials, Int. Math. Res. Not. 2012 (2012), 1699-1747.

[36] Kreiss H.-O., Initial boundary value problems for hyperbolic systems, Comm. Pure Appl. Math. 23 (1970), $277-298$.

[37] Krichever I.M., An analogue of the d'Alembert formula for the equations of a principal chiral field and the sine-Gordon equation, Dokl. Akad. Nauk SSSR 253 (1980), 288-292.

[38] Paley R.E.A.C., Wiener N., Fourier transforms in the complex domain, American Mathematical Society Colloquium Publications, Vol. 19, Amer. Math. Soc., Providence, RI, 1987.

[39] Sabatier P.C., Elbow scattering and inverse scattering applications to LKdV and KdV, J. Math. Phys. 41 (2000), 414-436.

[40] Sabatier P.C., Generalized inverse scattering transform applied to linear partial differential equations, Inverse Problems 22 (2006), 209-228.

[41] Sakhnovich A.L., The N-wave problem on the half-line, Russ. Math. Surv. 46 (1991), no. 4, $198-200$.

[42] Sakhnovich A.L., The Goursat problem for the sine-Gordon equation, and an inverse spectral problem, Russ. Math. Iz. VUZ (1992), no. 11, 42-52.

[43] Sakhnovich A.L., Second harmonic generation: Goursat problem on the semi-strip, Weyl functions and explicit solutions, Inverse Problems 21 (2005), 703-716, nlin.SI/0402055.

[44] Sakhnovich A.L., Weyl functions, the inverse problem and special solutions for the system auxiliary to the nonlinear optics equation, Inverse Problems 24 (2008), 025026, 23 pages, arXiv:0708.1112.

[45] Sakhnovich A.L., On the compatibility condition for linear systems and a factorization formula for wave functions, J. Differential Equations 252 (2012), 3658-3667.

[46] Sakhnovich A.L., Inverse problem for Dirac systems with locally square-summable potentials and rectangular Weyl functions, J. Spectr. Theory 5 (2015), 547-569, arXiv:1401.3605. 
[47] Sakhnovich A.L., Nonlinear Schrödinger equation in a semi-strip: evolution of the Weyl-Titchmarsh function and recovery of the initial condition and rectangular matrix solutions from the boundary conditions, J. Math. Anal. Appl. 423 (2015), 746-757.

[48] Sakhnovich A.L., Sakhnovich L.A., Roitberg I.Ya., Inverse problems and nonlinear evolution equations. Solutions, Darboux matrices and Weyl-Titchmarsh functions, De Gruyter Studies in Mathematics, Vol. 47, De Gruyter, Berlin, 2013.

[49] Sakhnovich L.A., Evolution of spectral data, and nonlinear equations, Ukrain. Math. J. 40 (1988), $459-461$.

[50] Sakhnovich L.A., Integrable nonlinear equations on the semi-axis, Ukrain. Math. J. 43 (1991), 1470-1476.

[51] Sakhnovich L.A., The method of operator identities and problems in analysis, St. Petersburg Math. J. 5 (1994), 1-69.

[52] Sakhnovich L.A., Spectral theory of canonical differential systems. Method of operator identities, Operator Theory: Advances and Applications, Vol. 107, Birkhäuser Verlag, Basel, 1999.

[53] Seeley R.T., Classroom notes: Fubini implies Leibniz implies $F_{y x}=F_{x y}$, Amer. Math. Monthly 68 (1961), $56-57$.

[54] Simon B., A new approach to inverse spectral theory. I. Fundamental formalism, Ann. of Math. 150 (1999), 1029-1057, math.SP/9906118.

[55] Sklyanin E.K., Boundary conditions for integrable equations, Funct. Anal. Appl. 21 (1987), 164-166.

[56] Teschl G., Jacobi operators and completely integrable nonlinear lattices, Mathematical Surveys and Monographs, Vol. 72, Amer. Math. Soc., Providence, RI, 2000.

[57] Ton B.A., Initial boundary value problems for the Korteweg-de Vries equation, J. Differential Equations 25 (1977), 288-309.

[58] Zakharov V.E., Manakov S.V., The theory of resonance interaction of wave packets in nonlinear media, Soviet Phys. JETP 69 (1975), 1654-1673.

[59] Zakharov V.E., Shabat A.B., Exact theory of two-dimensional self-focusing and one-dimensional selfmodulation of waves in nonlinear media, Soviet Phys. JETP 61 (1971), 62-69.

[60] Zakharov V.E., Shabat A.B., Integration of nonlinear equations of mathematical physics by the method of the inverse scattering problem. II, Funct. Anal. Appl. 13 (1979), 166-174. 